\title{
Fatter or fitter? On rewarding and training in a contest
}

\author{
Derek J. Clark $^{1}$ (D) Tore Nilssen $^{2}$ (i)
}

\begin{abstract}
${ }^{1}$ School of Business and Economics, UiT The Arctic University of Norway, Tromsø, Norway

${ }^{2}$ Department of Economics, University of Oslo, Oslo, Norway
\end{abstract}

\section{Correspondence}

Derek J. Clark, School of Business and Economics, UiT - The Arctic University of Norway, Tromsø NO-9037, Norway.

Email: derek.clark@uit.no

\begin{abstract}
Competition between heterogeneous participants leads to low-effort provision in contests. A principal can divide her fixed budget between skill-enhancing training and the contest prize. Training can reduce heterogeneity, increasing effort. It also reduces the contest prize, making effort fall. We set up an incomplete-information contest with heterogeneous players and show how this trade-off is related to the size of the budget of an effort-maximizing principal. A selection problem arises implying a cost associated with a win by the inferior player. The principal has a larger incentive to train the laggard, reducing the prize on offer.
\end{abstract}

K E Y W O R D

budget division, contest, selection problem, skill-enhancement

J E L C L A S S I F I C A T I O N

D74, D72, D82

\section{1 | INTRODUCTION}

How should an employer get the most out of her work force? Similarly, how should a research council get the most out of researchers? The standard answer in many such contexts is: set up a contest with a prize to the winner - like a promotion or a research grant. But, as many contest organizers have observed, contests do not incentivize well when there are big differences among the contestants at the outset. ${ }^{1}$ So the modified answer is: set up a contest, and seek to level the playing field among the contestants. ${ }^{2}$ However, what if leveling the playing field is costly? In such cases, the contest organizer might have to trade off the prize to the winner with spending resources on training the contestants so that they are both better equipped to put in effort in the contest and more interested in doing so.

We address the question of how to find the best balance between prize and training in a setting where a principal organizing a contest has a fixed budget that she can split between a prize, which will incentivize the contestants to put in more effort, and skill-improving training, which will make the effort put in by a contestant more productive. When there are ex-ante differences in the contestants' skills, there is also a question of who to train.

In our model, there are two contestants who compete in an all-pay auction, meaning that the winner is the contestant with the higher effort. A contestant's ex-ante skills are not known by the other contestant, nor by the principal. But everybody knows the probability distribution that each contestant's skill is drawn from. The ex-ante leader is the one with skills drawn from the better distribution, while the other one is called the ex-ante laggard.

When the principal aims at maximizing the contestants' total expected efforts, it turns out that the exact nature of her decision on how to split her budget between prize and training depends on how large the budget is. With a medium-

This is an open access article under the terms of the Creative Commons Attribution License, which permits use, distribution and reproduction in any medium, provided the original work is properly cited.

(c) 2021 The Authors. Economic Inquiry published by Wiley Periodicals LLC on behalf of Western Economic Association International. 
sized budget, the principal spends resources on training the laggard exactly so that any ex-ante differences are evened out, with the rest of the budget being spent on the prize. When the budget is small, the budget is optimally split between the prize and training of the ex-ante laggard, but such that the ex-ante difference is not totally evened out; and if the budget is very small, there will be no training and the whole budget is spent on the prize. When the budget is large, there is room for training both contestants in such a way that the ex-ante difference is first evened out, and then the expected abilities of both contestants are increased symmetrically, while still having funds for a prize.

We also discuss the case where the principal cares about having the right winner of the contest. Since skills are uncertain ex-ante, there is a chance that the winner is not the ex-post more efficient contestant. In order to take care of this problem, the principal should aim at minimizing the probability of erroneous selection. Interestingly, the size of the prize plays no role in this problem, so the only remaining issue is how to split the training part of the budget between the two contestants. We show that the probability of erroneous selection is not monotonic in the amount of training given.

In addition to simply minimizing the probability of selecting the less efficient contestant, we also consider the case in which the principal cares about the expected cost of erroneous selection (as measured by the difference in ability between the winner and the more efficient loser). Maximizing a weighted combination of expected effort minus expected selection cost, we show that the principal is more likely to offer training to the laggard for lower budgets, the less weight is placed on effort.

Our analysis provides insights for many situations where a principal is faced with heterogeneity among her contestants. We will briefly mention two such situations that are of particular importance.

For a country's research and technology policy, it is important to allocate public funds to researchers in the most efficient way possible. There has been a concern, at least since Merton (1968), that there is too much heterogeneity among research groups and that this leads to a lack of interest among researchers in taking part in competitions for funds from the research council. A question arises whether funds should be given directly to universities and other research institutions, in such a way that laggards become more able to compete for further funding, or should be deployed through grant competitions organized by the research council. As our analysis shows, the role for the research council is larger the smaller is the total budget allocated to research funding. As this budget increases, more of it should be allocated to leveling the playing field among the country's research institutions.

Another equally important topic is how firms should allocate their human-resource funds. In this research area, there are discussions whether one should focus on selecting the best workers for promotions to more prestigious and/or interesting jobs or on training the whole workforce in order to make them better able to compete for the promotions; see, for example, Beck-Krala (2020), Cron et al. (2005), and Farrell and Hakstian (2001). Training has been given special attention in sales force management, and many companies use substantial resources on this activity (see, e.g., Krishnamoorthy et al., 2005). ${ }^{3}$ Among others, Beck-Krala (2020) notes a tendency in human resource management to consider the "total reward" of an employee, encompassing financial and nonfinancial, direct and indirect rewards. Training is one costly dimension of the total reward offered by a firm. Our analysis shows that incentivizing the workforce through promotion contests and the like should be the main focus when the firm's funds are limited, whereas a richer firm can focus more on training the workforce in order to rectifying biases and in this way make the whole workforce interested in putting in efforts to obtain positions within the firm. ${ }^{4}$

Our paper builds on earlier discussions of all-pay auctions where players have private information about their valuations, such as Amann and Leininger (1996) and Clark and Riis (2001). In particular, Clark and Riis (2001) are close to our basic framework, since they posit two players where one has its skill drawn from a more advantageous distribution than the other, so that they, ex-ante, are leader and laggard. See also Seel (2014), where the private information is one-sided, in that one player's valuation is known by both players.

This paper is related to the discussion of whether and how to rectify ex-ante biases among contestants. See, in particular, Franke et al. (2018), Kirkegaard (2012), and Li and Yu (2012) for discussions on how to increase total effort by rectifying these biases. ${ }^{5}$ Our present analysis differs from the previous work in insisting that favoring a contestant is costly and will, in the face of a fixed total budget for the contest designer, imply a lower prize for the contest winner. In fact, we find that, when the budget is small, it is not optimal for the contest designer to completely rectify the ex-ante biases, since doing so would leave a too small prize to the winner. ${ }^{6}$

A number of studies discuss contest design when the designer has other concerns than simply maximizing total expected efforts. ${ }^{7}$ Tsoulouhas et al. (2007) discuss a designer facing one group of contestants with known abilities and another group with unknown abilities and find that it may be optimal to favor the former group. Cohen et al. (2008) discuss a designer who has an interest in maximizing the highest expected effort of a player and find that ex-ante biases are preferable. In Seel and Wasser (2014), the designer has concerns for both total expected efforts and the highest 
expected effort, and again having a bias is optimal. Pérez-Castrillo and Wettstein (2016) study a designer who wants to select the high-type contestant and find conditions such that, also in this case, having an ex-ante bias is preferable. Our analysis, in contrast, shows that, for a sufficiently large budget, it is optimal for the designer to level the playing field irrespective of how much weight she puts on selecting the high-type contestant, while for smaller budgets, a high such weight means a higher concern for training the laggard in order to reduce the ex-ante bias.

The paper relates to studies of pre-contest investments. For example, Fu and $\mathrm{Lu}$ (2009) and Konrad (2002) discuss contestants' incentives to invest in own productivity before a contest. In contrast, we discuss the principal's incentives for such pre-contest investments. Clark and Nilssen (2013) analyze contestants' incentives to put in extra effort in the first round of a two-round competition where there is complete information and learning by doing. They discuss how the contest designer can split her prize budget across the two rounds in order to get the right balance between firstround learning and second-round efforts. This is related to the present discussion of pre-contest training versus prize award; the framework of the present paper is quite different, however, since heterogeneous players compete under incomplete information, and the ex-post effect of training is not deterministic.

The paper is organized as follows. Section 2 outlines the basic contest played between heterogeneous participants. Section 3 considers how an effort maximizing principal will divide her budget between training and the contest prize. Section 4 focuses on the selection problem in which a low-ability contestant can win the contest; the trade-off between the prize and training is considered here for a principal that maximizes a weighted sum of the expected contest effort and the expected cost of erroneous selection. Section 5 concludes. All proofs are to be found in Appendix A.

\section{2 | THE CONTEST}

Two risk neutral players compete for a prize of size $v$ by exerting irreversible efforts $x_{i} \geq 0, i=1,2$. The cost of effort to player $i$ is given by $\frac{x_{i}}{a_{i}}$, where $a_{i}$ is an ability parameter that is private information to that player. It is commonly known that player 1 draws ability from a uniform distribution on $[h, H]$, and player 2 from a uniform distribution on $[l, L]$. We make the following assumption:

Assumption 1 (i) $H-h=L-l \equiv D$. (ii) $H \geq L>h \geq l>0$. (iii) $\frac{H}{D}>\frac{4}{3}$.

Part (i) of the assumption implies that the players' distributions are identical up to a location shift. Part (ii) means that player 1, without loss of generality, is expected to be the more able player ex ante, with $H \geq L$. It also implies that the ability distributions are overlapping, with $L>h$, which again implies that $D>H-L$; and that $L>D$, since $l=L-D>0$. Expost it can hence be the case that player 2 is actually more able, even though player 1 is expected to have the higher ability ex ante. Part (iii) is a regularity assumption. It is not a very strong assumption to make. Necessarily, $\frac{H}{D}>1$, since $h=H-D>0$. Suppose, moreover, that $L$ approaches $h$, which would mean that $h-l$ would approach $D$. With $L-l=D$ and $l>0$, this would imply $h>D$, or, since $h=H-D, \frac{H}{D}>2$, which is stricter than the assumption we make here.

The player with the larger effort wins the prize with certainty, with ties broken randomly, as depicted by the following contest success function giving the probability that player 1 wins the prize:

$$
p_{1}\left(x_{1}, x_{2}\right)=\left\{\begin{array}{l}
1 \text { if } x_{1}>x_{2} \\
\frac{1}{2} \text { if } x_{1}=x_{2} \\
0 \text { if } x_{1}<x_{2}
\end{array}\right.
$$

At the contest stage, player $i$ knows his own ability but not the ability of the opponent. The expected payoffs of type $a_{i}$ can be written as

$$
\begin{aligned}
& \pi_{1}\left(x_{1}, x_{2}, a_{1}\right)=\left(\operatorname{Pr}\left(x_{1}>x_{2}\right)+\frac{1}{2} \operatorname{Pr}\left(x_{1}=x_{2}\right)\right) v-\frac{x_{1}}{a_{1}} \\
& \pi_{2}\left(x_{1}, x_{2}, a_{2}\right)=\left(\operatorname{Pr}\left(x_{2}>x_{1}\right)+\frac{1}{2} \operatorname{Pr}\left(x_{1}=x_{2}\right)\right) v-\frac{x_{2}}{a_{2}}
\end{aligned}
$$




\section{$4 \_$Economic Inpuiry}

Let the effort function $x_{i}\left(a_{i}\right)$ of player $i$ be a mapping from the player's ability to his chosen effort; suppose it is continuous and strictly increasing (except possibly at zero), which implies that there exists an inverse $g_{i}\left(x_{i}\right)=x_{i}^{-1}\left(x_{i}\right)=a_{i}$, $i=1,2$. Since abilities are uniformly distributed, we can write expected payoffs for the two players as

$$
\begin{aligned}
& \pi_{1}\left(x_{1}, x_{2}, a_{1}\right)=\frac{g_{2}\left(x_{1}\right)-l}{D} v-\frac{x_{1}}{a_{1}} \\
& \pi_{2}\left(x_{1}, x_{2}, a_{2}\right)=\frac{g_{1}\left(x_{2}\right)-h}{D} v-\frac{x_{2}}{a_{2}}
\end{aligned}
$$

Using arguments explained in Clark and Riis (2001), we can state the following result, the proof of which is in the Appendix.

Proposition 1 The unique pure-strategy Bayesian Nash equilibrium is given by the equilibrium effort functions $x_{i}^{*}\left(a_{i}\right), i=1,2$, where

$$
\begin{aligned}
& x_{1}^{*}\left(a_{1}\right)=\frac{L\left(a_{1}^{2}-h^{2}\right)}{2 D H} v, \text { for } a_{1} \in[h, H] . \\
& x_{2}^{*}\left(a_{2}\right)= \begin{cases}0, & \text { for } a_{2} \in\left[l, \frac{L h}{H}\right] ; \\
\frac{a_{2}^{2} H^{2}-L^{2} h^{2}}{2 D H L} v, & \text { for } a_{2} \in\left(\frac{L h}{H}, L\right] .\end{cases}
\end{aligned}
$$

Whilst almost all player- 1 types have positive effort, some low player-2 types $\left(a_{2} \in\left[l, \frac{L h}{H}\right]\right)$ do not find it worthwhile to compete. Note that the two players' equilibrium effort functions have the same support, $[\underline{x}, \bar{x}]=\left[0, \frac{L}{H}\left(H-\frac{D}{2}\right)\right]$. Note also that, when the players draw their valuations from the same uniform distribution (i.e., when $L=H$ ), the equilibrium effort functions are

$$
x_{i}^{*}\left(a_{i}\right)=\frac{a_{i}^{2}-h^{2}}{2 D} v, i=1,2 .
$$

Figure 1 gives an illustration of the equilibrium in Proposition 1, showing that the equilibrium effort function of the ex-ante less able player 2 lies over that of player 1. The superior opponent uses his expected edge to slack off and save on effort cost. This means that a player- 2 type of inferior ability can beat a more able player- 1 type. When the players have drawn the same ability $a_{1}=a_{2}=a$, which of course can only happen if $a>h$, it is easy to verify from Equations (3) and (4) that $x_{2}^{*}(a)>x_{1}^{*}(a)$ when $H>L$.

The ex-ante total expected efforts (i.e., before the draws are made) are

$$
\begin{aligned}
X^{*} & =E\left(x_{1}^{*}\right)+E\left(x_{2}^{*}\right) \\
& =\frac{L v}{2 D H} \int_{h}^{H}\left(a_{1}^{2}-h^{2}\right) \frac{1}{D} d a_{1}+\frac{v}{2 D H L} \int_{\frac{L h}{H}}^{L}\left(a_{2}^{2} H^{2}-L^{2} h^{2}\right) \frac{1}{D} d a_{2} \\
& =\frac{L(3 H-2 D)}{6 H} v+\frac{L^{2}(3 H-2 D)}{6 H^{2}} v \\
& =\frac{L(3 H-2 D)(H+L)}{6 H^{2}} v,
\end{aligned}
$$

where we use the substitution $h=H-D$.

Note that the ratio of the expected efforts has a simple form: 




F I G U R E 1 Equilibrium effort functions

$$
\frac{E\left(x_{1}^{*}\right)}{E\left(x_{2}^{*}\right)}=\frac{H}{L} \geq 1
$$

Thus, even if, for a given $a$, player 2 has the higher effort, the ex-ante expected effort is higher for player 1. Moreover, in the case of symmetry, when $L=H$, the expression in Equation (7) reduces to

$$
\left(H-\frac{2}{3} D\right) v
$$

Player 1 wins the contest with certainty if player 2 draws a type in the interval $\left[l, \frac{L h}{H}\right]$, since 2 then has zero effort in equilibrium; player 1 also wins if $x_{1}^{*}\left(a_{1}\right)>x_{2}^{*}\left(a_{2}\right)$, which by Proposition 1 occurs for $a_{2}<\frac{L}{H} a_{1}$. Hence, the probability that player 1 of type $a_{1}$ wins is $\frac{1}{D}\left(\frac{a_{1} L}{H}-l\right)$; taking the expectation of this over all player-1 types gives the ex-ante probability that player 1 wins the contest in equilibrium as ${ }^{8}$ :

$$
p_{1}^{*}=\int_{h}^{H} \frac{1}{D}\left(\frac{a_{1} L}{H}-l\right) \frac{1}{D} d a_{1}=1-\frac{L}{2 H} \geq \frac{1}{2}
$$

where the inequality follows from $L \leq H$.

Even though the ex-ante more able player 1 is expected to have more effort, this does not cost him more, since his unit cost of effort is likely to be smaller. In fact, the expected ex-ante costs of effort of the two players are identical:

$$
\int_{h}^{H} \frac{x_{1}^{*}\left(a_{1}\right)}{a_{1}} \frac{1}{D} d a_{1}=\int_{\frac{L h}{H}}^{L} \frac{x_{2}^{*}\left(a_{2}\right)}{a_{2}} \frac{1}{D} d a_{2}=\frac{L v}{4 H D^{2}}\left[D(2 H-D)-2 \ln \frac{H}{H-D}\right] .
$$

The ex-ante expected payoffs to the players can be found as

$$
\begin{aligned}
E \pi_{1}^{*} & =\left(1-\frac{L}{2 H}\right) v-\frac{L v}{4 H D^{2}}\left[D(2 H-D)-2 \ln \frac{H}{H-D}\right] ; \\
E \pi_{2}^{*} & =\left(\frac{L}{2 H}\right) v-\frac{L v}{4 H D^{2}}\left[D(2 H-D)-2 \ln \frac{H}{H-D}\right] \\
& =\frac{L v}{4 H D^{2}}\left[D(3 D-2 H)+2(H-D)^{2}\left(\ln \frac{H}{H-D}\right)\right]:=\frac{L v}{4 H D^{2}} \Theta(D, H) ;
\end{aligned}
$$


where the expected payoff to player 1 is higher, since he has the higher win probability in equilibrium and the players have the same expected cost of effort. Player 2 must achieve a nonnegative profit in equilibrium, that is, $\Theta(D, H) \geq 0$. It is easily verified that $\Theta(0, H)=0$, and that it is positive otherwise so that the expectedly weakest player has a nonnegative payoff in equilibrium as required.

\section{3 | TRAINING TO MAXIMIZE EFFORT}

Suppose the principal aims at maximizing the total ex-ante expected efforts of the contestant. She has available a fixed budget $B$, which can be divided between giving the contest prize $v$ and investing in the abilities of the players with $s_{1} \geq 0$ and $s_{2} \geq 0$, respectively. Budget balance requires $B=v+s_{1}+s_{2}$.

The development of ability at the training stage is modeled as an upward shift in the ability interval of the receiving player, keeping the length of the interval constant at $D$. With expenditure $s_{i}$, the ability improvement is simply $s_{i}$; following expenditures of $s_{1}$ and $s_{2}$ on the two players, the ability interval of player 1 becomes $\left[h+s_{1}, H+s_{1}\right]$, while player 2 has $\left[l+s_{2}, L+s_{2}\right]$.

At the beginning of the game, the principal announces a triple $\left(v, s_{1}, s_{2}\right)$ that satisfies budget balance. If either of the training amounts is positive, then training takes place. Then draws are made from the modified ability distributions. After this the contest is played over the prize $v$.

The equilibrium is based on the premise that (a) the ability intervals overlap, and (b) that the ability intervals are of the same length with that of player 1 being above that of the rival. To ensure that the ability intervals overlap after any training is carried out requires that $L+s_{2}>h+s_{1}=H-D+s_{1}$; after training, player 1 retains the higher ability interval as long as $H+s_{1}>L+s_{2} \cdot{ }^{9}$ Training levels that fulfill both of these requirements hence satisfy

$$
H-L-D<s_{2}-s_{1}<H-L .
$$

To facilitate comparative-statics analysis when the lower and upper bounds of the interval are changed, it is convenient to rewrite the equilibrium effort functions in Equations (3) and (4) using $h=H-D, l=L-D$, since $D$ is constant. We have

$$
\begin{aligned}
& x_{1}^{*}\left(a_{1}\right)=\frac{L\left[a_{1}^{2}-(H-D)^{2}\right]}{2 D H} v, \text { for } a_{1} \in[H-D, H] . \\
& x_{2}^{*}\left(a_{2}\right)= \begin{cases}0, & \text { for } a_{2} \in\left[L-D, \frac{L(H-D)}{H}\right] ; \\
\frac{a_{2}^{2} H^{2}-L^{2}(H-D)^{2}}{2 D H L} v, & \text { for } a_{2} \in\left(\frac{L(H-D)}{H}, L\right] .\end{cases}
\end{aligned}
$$

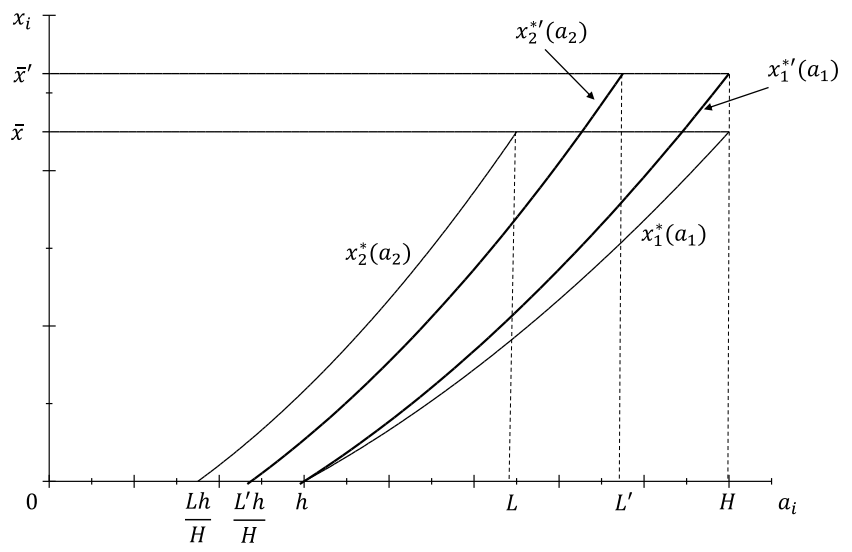

F I G U R E 2 Equilibrium efforts when player 2 receives training 


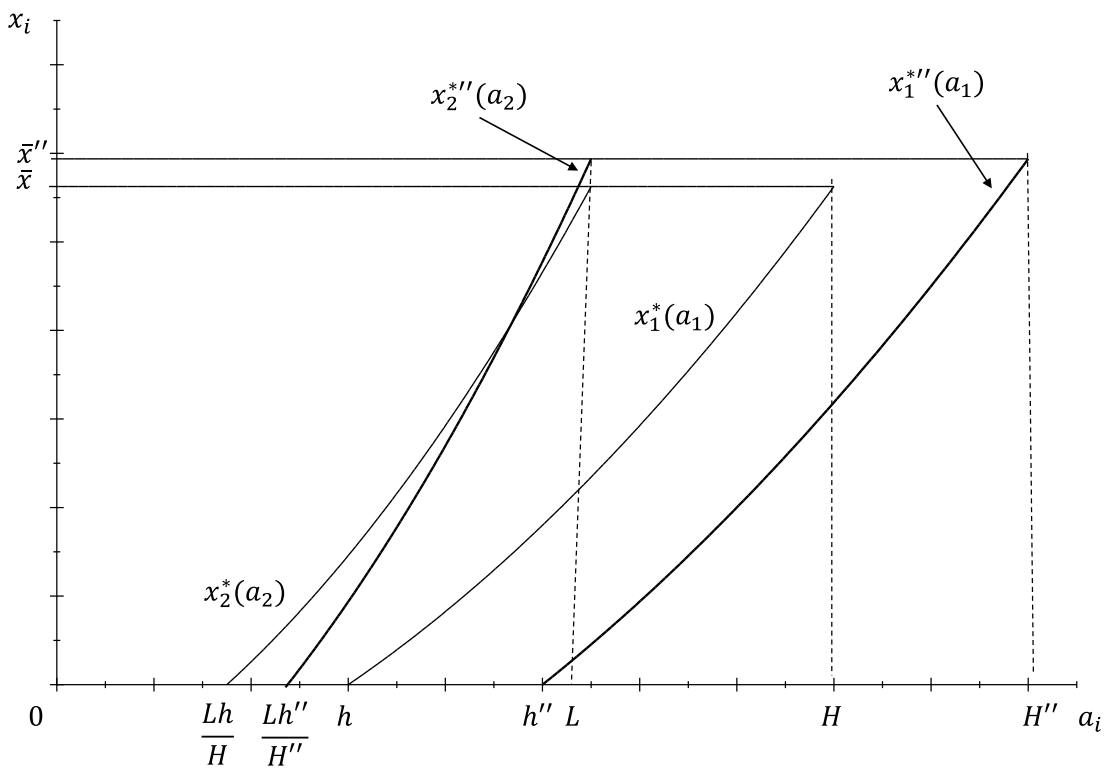

F I G U R E 3 Efforts when player 1 is trained

We can now analyze the effect that increasing the expected ability of one of the players will have on the equilibrium effort functions. Suppose first that the support of the distribution for the laggard is moved up (i.e., $l$ and $L$ increase). The effect that this has on the equilibrium effort functions is drawn in Figure 2 for a shift from $L$ to $L^{\prime}>L$, where the new equilibrium efforts are given by $x_{i}^{* \prime}\left(a_{i}\right) .{ }^{10}$

From this, it is apparent that the effects on the effort functions are monotonic; all player- 1 abilities increase their efforts, since the rival is now expected to be more able than before. The laggard responds to the expected increase in ability by providing less effort. On the other hand, the high player-2 types will have effort above the previous maximum level. The common upper support of both players increases to $\bar{x}^{\prime}$.

Figure 3 depicts the effects of increasing the expected ability of the leader, that is, increasing $(h, H)$ to $\left(h^{\prime \prime}, H^{\prime \prime}\right) .{ }^{11}$

Whilst the response of the receiving player 1 is to lower effort for all ability levels, except at the top of the distribution, the response of player 2 is to decrease effort for low ability levels and increase it for high ones. There are also fewer player 2 types that have positive effort when the opponent becomes more superior in expectation.

The principal knows that player 1 is the expectedly more able; since she does not know the actual draws made by the rivals, the principal does not know which of the players is most able ex-post. As illustrated in Figures 2 and 3, increasing the expected ability of the laggard causes the effort function of the leader to shift upwards and that of the laggard to shift downward; increasing the expected ability of the leader reduces the efforts of that player and of low laggard types, but increases the effort of higher ability laggards.

Dividing the budget between the prize and training for one of the players is not a straightforward problem as demonstrated above. The problem becomes more complex when both can receive training. However, as it turns out, the principal will not support the ex-ante leader with any training in our model, except if the budget is large, so that the optimum is to split the budget between a prize to the contest winner and training of the ex-ante laggard. In particular, we have:

Proposition 2 A principal with a budget of B will split the budget on prize and training as follows:

(i) An insufficient budget, that is, one where

$$
0<B \leq \frac{L(H+L)}{H+2 L}
$$

will lead to no training and $v=B$.

(ii) If the budget is small, that is, if

$$
\frac{L(H+L)}{H+2 L}<B \leq \frac{5}{3} H-L
$$




\section{$8 \_$Economic Inpuiry}

then the principal spends $s_{2}$ on training the ex-ante laggard and the rest, $B-s_{2}$, on the prize, where

$$
s_{2}=\frac{1}{3}\left(B-H-2 L+\sqrt{(B+H+L)^{2}-H(B+L)}\right) .
$$

(iii) If the budget is of an intermediate size, that is, if

$$
\frac{5}{3} H-L<B \leq 3 H-L-\frac{4}{3} D
$$

then the principal trains the ex-ante laggard until the two players have equal expected skills,

$$
s_{2}=H-L,
$$

and uses the rest, $B-H+L$, as the contest prize.

(iv) If the budget is large, that is, if

$$
B>3 H-L-\frac{4}{3} D
$$

then the principal first spends training on the ex-ante laggard until the two players have equal expected skills, and thereafter spends equal amount of training on both players so that they continue to have equal expected skills. Total spending on training is $S=s_{1}+s_{2}$, while the rest of the budget, $B-S$, is spent on the prize, where

$$
\begin{aligned}
S & =\frac{1}{6}(3 B-3 H-3 L+4 D) \\
s_{1} & =\frac{S-(H-L)}{2} \\
s_{2} & =\frac{S+(H-L)}{2} .
\end{aligned}
$$

Consider first part (i), which indicates the case in which training is completely sacrificed in order to give a contest prize as large as possible. When the principal is resource constrained in this way, training the laggard has a positive effect on total effort ceteris paribus, but this directly reduces the contest prize, reducing effort. The second effect outweighs the first, and no training is given.

Part (ii) covers the case when the budget is larger, but not enough that it pays to make the players symmetric. Now total efforts get a boost from the laggard being trained, at the same time as there is a downward pressure on total efforts as the prize becomes lower and lower. The amount of the budget used on training balances these two effects, finding an internal division of the budget. Increasing the budget further, as in part (iii), allows the laggard to be trained until the contestants are equal in expected ability, putting the remainder of the budget into the prize fund. Finally, in part (iv), the budget is so large that the initial laggard can be trained so that he catches up the expected leader, and then both players can be made more efficient. This occurs until the marginal effect of spending one unit of the budget on training is equal to the marginal effect of giving that unit as a prize.

The relationship between the size of the budget and the total expected efforts is then straightforward to determine as

$$
X^{*}= \begin{cases}\frac{L(3 H-2 D)(H+L)}{6 H^{2}} B, & \text { for } 0<B \leq \frac{L(H+L)}{H+2 L} \\ \frac{(3 H-2 D)(\Psi-H)(2 H+\Psi)(3 B+H+3 L-\Psi)}{162 H^{2}}, & \text { for } \frac{L(H+L)}{H+2 L}<B \leq \frac{5}{3} H-L ; \\ \left(H-\frac{2}{3} D\right)(B-H+L), & \text { for } \frac{5}{3} H-L<B \leq 3 H-L-\frac{4}{3} D \\ \frac{(3 B+3 H+3 L-4 D)^{2}}{72}, & \text { for } B>3 H-L-\frac{4}{3} D\end{cases}
$$


where $\Psi:=B+L+\sqrt{(B+H+L)^{2}-H(B+L)}$. The second and fourth parts of this function are increasing and convex in $B$. For very small and for intermediate budget sizes, the first and third parts indicate that extra budget is completely given to the prize, increasing expected effort linearly. This relationship between $B$ and $X^{*}$ is illustrated in Figure $4 .{ }^{12}$ To give an idea of the effect of training on expected effort, the line $X_{0}^{*}$ indicates expected effort without training, that is, $v=B, s_{1}=s_{2}=0 .^{13}$

For low budget levels, all funds are spent on the contest prize, and expected efforts are a fixed proportion of this, as indicated by Equation (7). When the budget reaches $\frac{L(H+L)}{H+2 L}$, it is possible to do better than this by training the laggard. Figure 2 shows that the leader will increase effort for all ability types but that the laggard will reduce effort, apart from the high types that are created by the training. Initially, as the budget increases beyond $\frac{L(H+L)}{H+2 L}$, the net effect is positive and large enough to outweigh the fact that resources are taken away from the contest prize, which reduces effort. As further resources are used on training player 2, the players become more and more alike in expected ability; this leveling of the playing field increases effort. If the principal has a total budget of $\frac{5}{3} H-L$, then training is given until the players are symmetric; hence, $H-L$ is used on training player 2 , and $\frac{2 H}{3}$ is the contest prize. An increase in the budget from this point will optimally be put in its entirety into the prize fund; however, when the budget becomes large enough $\left(3 H-L-\frac{4}{3} D\right)$, some resources are allocated to training both players, keeping them symmetric and increasing their ability in the contest. This leads to an increase in expected effort that is larger than would be obtained simply by granting a larger prize.

In dividing the budget between the contest prize and training player 2, the principal imparts several effects on the expected payoffs of the players. In Figure 5, the ex-ante expected payoffs of the players when training is not given are represented by $E \pi_{1}^{0}$ and $E \pi_{2}^{0}$. Since each extra unit of budget gives an equivalent increase in the contest prize, these are straight lines and player 1 - who is expected to be most able-has the larger expected payoff. For low-budget levels (up to $\left.\frac{L(H+L)}{H+2 L}\right)$ there is no training and these basis payoffs are achieved by both players. When player 2 is trained, he expects to draw a lower cost of participating in the contest. As illustrated by Figure 2, this causes player 1 to exert more effort than before. This increases player 1's probability of winning, but it also increases its effort costs, so that player 1's expected payoff falls for budgets in $\frac{L(H+L)}{H+2 L}<B \leq \frac{5}{3} H-L$ - even when the principal also increases the contest prize in this region. As a response to training, most player-2 types slack off, which reduces the probability of this player winning. But less effort, exerted at a lower cost, and an increasing contest prize mitigates this. Figure 5 shows that the expected payoff of player 2 increases as the budget grows, even though it is lower than it would have been with no training. Note that the expected payoff to player 2 is convex in this interval. As the budget increases, more and more training is given, and player 2 reduces effort, but the marginal effect is smaller the more his ability interval is shifted upwards. Then the fact that effort becomes less and less expensive makes the payoff function slope up steeply, leading to a larger expected payoff than without training. In the interval $\frac{5}{3} H-L<B \leq 3 H-L-\frac{4}{3} D$, the ability intervals of both players are identical, and the ex ante expected payoff of both players is the same. It increases linearly in the budget since every extra dollar is given to the prize. When the budget is large, the principal decides to train both players symmetrically, so that only a fraction of the budget increase is given to the contest prize; hence the slope of the expected payoff function is reduced. It can be shown that the basis expected payoff of player $2\left(E \pi_{2}^{0}\right)$ is larger than the symmetric payoff with training for a sufficiently large budget. ${ }^{14}$

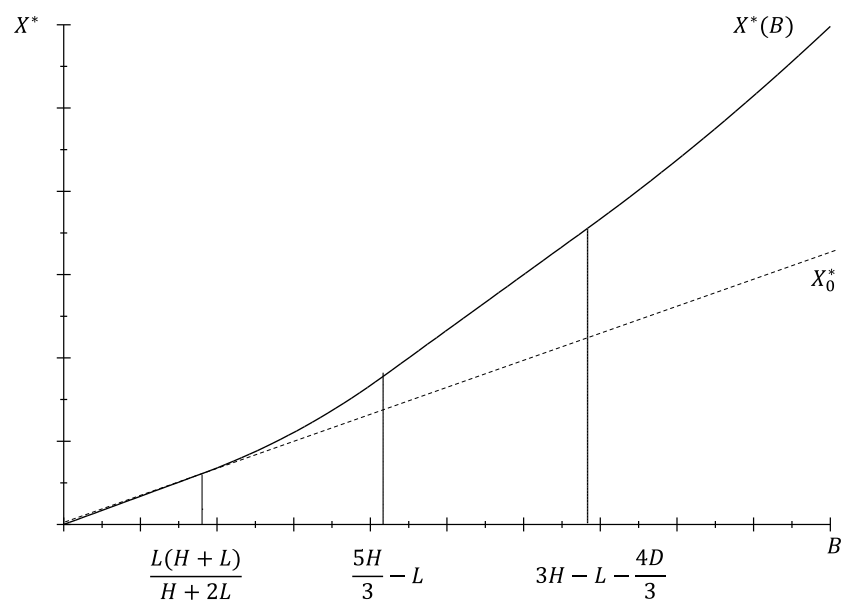

F I G U R E 4 Equilibrium efforts as a function of budget 


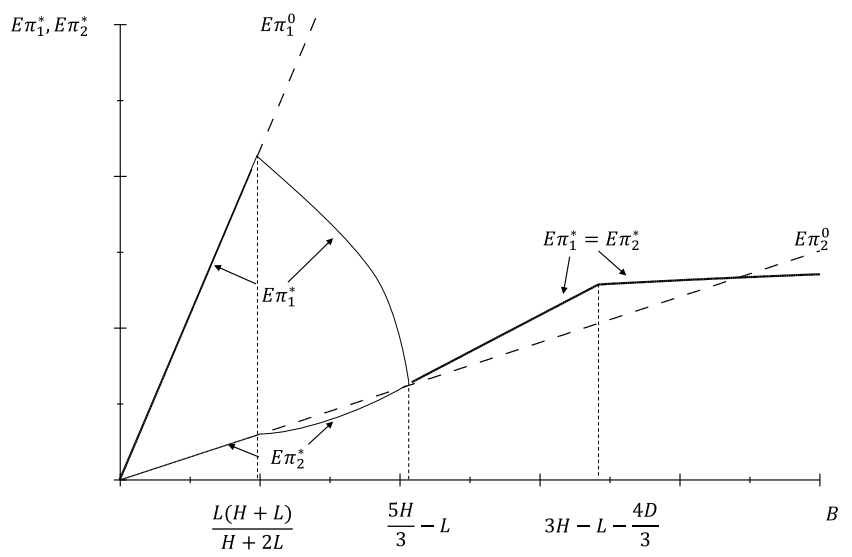

F I G U R E 5 Ex ante expected payoffs in equilibrium

\section{4 | ACCOUNTING FOR THE PROBLEM OF ERRONEOUS SELECTION}

In settings like the one we study here, it is not clear that the winner of the contest is the ex-post more efficient one: the ex-ante leader may draw a high skill but still end up losing because of the laggard's higher efforts. This ex-post selection problem - the problem of erroneous selection-is particularly important in settings such as promotion contests and competitions for research grants, where the winner goes on to perform tasks whose qualities may depend on the winner's skills. In this section, we therefore amend the principal's decision problem to incorporate a concern for erroneous selection. We do this by first studying a principal whose sole aim is to minimize the problem of erroneous selection and then use this analysis to study the general problem of a principal with an interest in both high total expected efforts and low expected costs of erroneous selection.

It is not possible for player 1 to win when player 2 is more able, thus we have no instance of a type-2 error. Player 1 wins when $a_{1}>\frac{H}{L} a_{2}$ and is more able in all such cases. We can calculate the probability of the principal making a type- 1 error ex-post, that is, the probability that contestant 2 wins when contestant 1 has the higher ex-post ability:

$$
\begin{aligned}
\rho^{*} & =\int_{L}^{H}\left(\frac{L-\frac{a_{1} L}{H}}{D}\right) \frac{1}{D} d a_{1}+\int_{h}^{L}\left(\frac{a_{1}-\frac{a_{1} L}{H}}{D}\right) \frac{1}{D} d a_{1} \\
& =\frac{H-L}{2 D^{2}}\left[L-\frac{(H-D)^{2}}{H}\right] .
\end{aligned}
$$

Note that the size of the prize $v$ does not affect $\rho^{*}$. Moreover, asymmetry (i.e., $H>L$ ) always leads to a positive probability of the contest selecting the player with the lower ability. ${ }^{15}$ The calculation of $\rho^{*}$ is demonstrated in Figure 6.

Player 1 wins the contest when abilities are in the areas marked by $A$ in the figure. In all these cases, we have that $a_{1}>a_{2}$, so that the more able is selected as winner. This is also the case for area $C$, where player 2 wins and is more able. The areas marked by $b$ and $b^{\prime}$ indicate combinations in which player 2 wins but is less able. The first element in Equation (15) represents area $b$, while the second one is $b^{\prime}$. When player 2 receives training, $L$ increases and the line $\frac{H}{L} a_{2}$ moves closer to the 45-degree line. This in itself reduces the areas $b$ and $b^{\prime}$. However, the square of feasible ability combinations shifts rightward in Figure 6, removing some low-ability player-2 types (who mostly lose to better player-1 types) and introducing some higher ability player- 2 types who can beat better opponents. Hence, the overall effect of training the laggard on the probability of erroneous selection is generally nonmonotonic. In fact, we can state the following result.

Proposition 3 (i) If $H$ is high and $L$ is low, in particular, if $H>\left(1+\frac{1}{\sqrt{2}}\right) D$, and $L \in\left(\max \{H-D, D\}, \frac{H}{2}+\frac{(H-D)^{2}}{2 H}\right)$, then $\frac{\partial \rho^{*}}{\partial L}>0$; otherwise, that is, if $H \in\left(\frac{4}{3} D,\left(1+\frac{1}{\sqrt{2}}\right) D\right)$ and/or $L \in\left(\frac{H}{2}+\frac{(H-D)^{2}}{2 H}, H\right)$, then $\frac{\partial \rho^{*}}{\partial L}<0$.

(ii) There exists an $\widehat{H}$ such that $\frac{\partial \rho^{*}}{\partial H}>0$ for $H \in(L, \widehat{H})$ and $\frac{\partial \rho^{*}}{\partial H}<0$ for $H \in(\widehat{H}, L+D)$. 


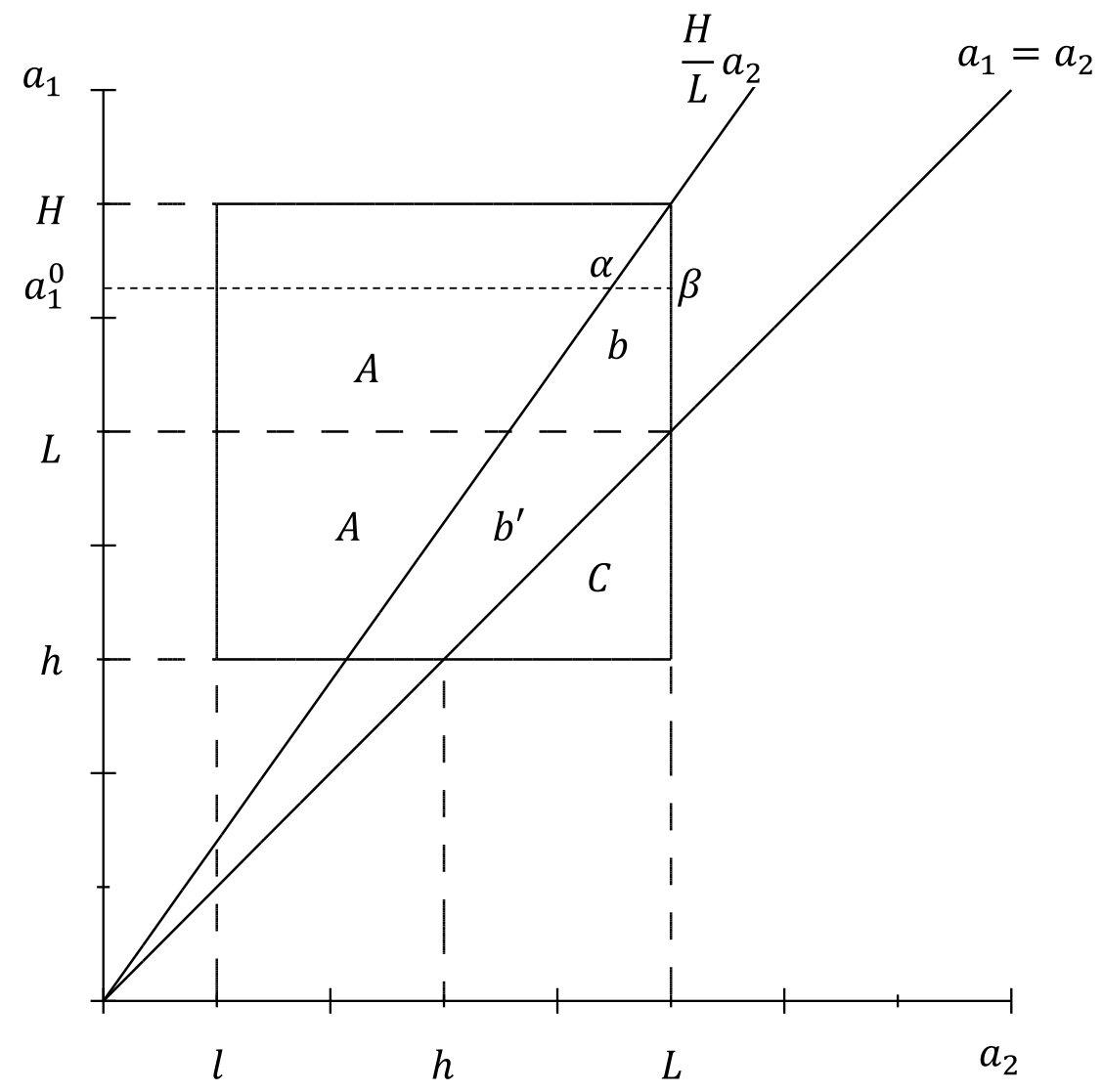

F I G U R E 6 Probability of erroneous selection

We see from part (i) of Proposition 3 that, when $H$ is sufficiently large, training player 2 by increasing $L$ can actually increase the probability of erroneous selection for low enough levels of $L$. In this range, training player 2 does not contribute to the contest picking the high-ability player. For higher values of $L$, on the other hand, training reduces the probability of picking the wrong winner. We also see that, for low values of $H$, training player 2 reduces the probability of picking the low-ability player as winner.

From part (ii), we see that training the ex-ante leader by increasing $H$ will increase the probability of erroneous selection in most cases. The exception is when $H$ is large, in which case further increases will lead to this probability falling, since the superior player 1 will win in most cases. The exact expression for $\widehat{H}$ is given in the proof of Proposition 3 in the Appendix.

Although we could think of minimizing $\rho^{*}$ as a way to deal with the selection problem, it is even better to let the principal put more weight on the type-1 error the bigger the difference between the contestants' ex-post abilities is - we can think of this as minimizing the expected cost of erroneous selection,

$$
\begin{aligned}
\Gamma^{*} & =\frac{1}{D^{2}}\left(\int_{L}^{H} \int_{\frac{a_{1} L}{H}}^{L}\left(a_{1}-a_{2}\right) d a_{2} d a_{1}+\int_{h}^{L} \int_{\frac{a_{1} L}{H}}^{a_{1}}\left(a_{1}-a_{2}\right) d a_{2} d a_{1}\right) \\
& =\frac{(H-L)^{2}}{6 D^{2}}\left[L-\frac{(H-D)^{3}}{H^{2}}\right] .
\end{aligned}
$$

Consider ability $a_{1}^{0}$ in Figure 6 . This ability type of player 1 loses to inferior player 2 types in the interval $a_{2} \in\left[\frac{L}{H} a_{1}^{0}, L\right]$, on the line segment $\alpha \beta$. The cost of losing to each opponent in this interval is $a_{1}-a_{2}>0$. Summing the expected cost over the interval gives $\frac{1}{D} \int_{\frac{a_{1} L}{H}}^{L}\left(a_{1}-a_{2}\right) d a_{2}$ as the expected cost of erroneous selection associated with player 1 type $a_{1}^{0}$. 
Summing over all player 1 types between $H$ and $L$ gives the expected cost of wrong selection associated with area $b$ in Figure 6. This is the first part of the bracketed expression in Equation (17). The second element in this equation sums up the costs associated with erroneous selection in area $b^{\prime}$.

A principal solely concerned with the ex-post selection problem will seek to minimize $\Gamma^{*}$. Note again that the prize $v$ plays no role in this problem. The expected cost of erroneous selection is 0 at $L=H$; while $\Gamma^{*}>0$ for $H>L$, since the square bracket in Equation (18) is positive for $L>\frac{(H-D)^{3}}{H^{2}}$, which holds. ${ }^{16}$ Contrary to the probability of erroneous selection, the expected cost $\Gamma^{*}$ is strictly monotonic in $L$ and $H$, as shown in the following Lemma.

Lemma 1 For $L<H$, (i) $\frac{\partial \Gamma^{*}}{\partial L}<0$; (ii) $\frac{\partial \Gamma^{*}}{\partial H}>0$. When $L=H$, $\frac{\partial \Gamma^{*}}{\partial L}=\frac{\partial \Gamma^{*}}{\partial H}=0$.

Given Lemma 1, we have the following:

Proposition 4 A principal who is solely concerned with minimizing the expected cost of erroneous selection will split the budget as follows:

(i) If $0<B \leq H-L$, then $v$ equals a small amount, while the rest of the budget is spent on training to get as close as possible to symmetry.

(ii) If $B>H-L$, then $s_{1}=0, s_{2}=H-L$, so that symmetry is obtained, and $v=B-H+L \geq 0$.

Since the amount of the prize does not affect the cost of erroneous selection, it is only needed in order to actually induce efforts in the contest. In part (i) of Proposition 4, the budget is not sufficient to achieve full symmetry between the players and hence a cost of wrong selection of zero; in this case, a small prize is given to ensure that efforts are positive so that the contest can work as a selection mechanism. The rest of the budget is used to get as close to symmetry as possible. Only when the budget is large enough to achieve full symmetry (Proposition 4, part [ii]) does the budget affect the size of the contest prize. In this case, the principal trains the laggard to full symmetry, and uses the residual budget as a prize. ${ }^{17}$

Consider next a principal who balances her concern for total expected efforts and that of the expected costs of erroneous selection. In particular, let her objective function be

$$
\begin{aligned}
\Omega^{*}(k) & =k X^{*}-(1-k) \Gamma^{*} \\
& =k \frac{L(3 H-2 D)(H+L)}{6 H^{2}} v-(1-k) \frac{(H-L)^{2}\left[H^{2} L-(H-D)^{3}\right]}{6 H^{2} D^{2}},
\end{aligned}
$$

where $k \in[0,1]$ is the weight put on total expected efforts. The cases of $\Omega^{*}(1)$ and $\Omega^{*}(0)$ are discussed above, with results presented in Propositions 2 and 4, respectively. There is a clear trade-off that balances the two parts of the objective function, since giving more prize increases contest effort, but leaves less for training, so that the cost of erroneous selection increases. Note from Proposition 2 that, when the budget is exactly $B=\frac{5}{3} H-L$, the principal optimally trains the laggard until the contestants are expectedly of equal skill, and hence there will be no selection cost. This means that, for $B=\frac{5}{3} H-L$, the principal sets $s_{2}=H-L, v=B-s_{2}=\frac{2}{3} H$, and this is independent of $k$. For budgets below this, the weight $k$ will affect the division between the contest prize and the training given. Again, it is optimal to only train the laggard $\left(s_{1}=0, s_{2} \geq 0\right)$, and we can show the following result:

Proposition 5 Let $k \in[0,1]$ be the weight the principal puts on total expected efforts. Let, for each $k$, $T(k)=\left(t(k), \frac{5}{3} H-L\right)$ denote a range of the nonnegative real line such that, if the principal's budget $B \in T(k)$, then the principal's decision to train the laggard is an interior solution $s_{2}(k) \in(0, H-L)$, so that the laggard receives some training, but not enough to capture the skill level of the leader. Then, in equilibrium, $\frac{d s_{2}}{d k}<0$, and $\frac{d t}{d k} \geq 0$, with $\frac{d t}{d k}>0$ whenever $t(k)>0$.

As shown in Proposition 2, the optimal budget division to maximize expected effort involves some training and some contest prize, except for very low budgets. Since the expected cost of erroneous selection is independent of the contest prize, lowering $k$ from 1 gives the principal an extra incentive to train the laggard, and this incentives becomes stronger as $k$ falls. Hence, the budget at which training starts $(t(k))$ is lower, the lower is $k$ except possibly for cases where 


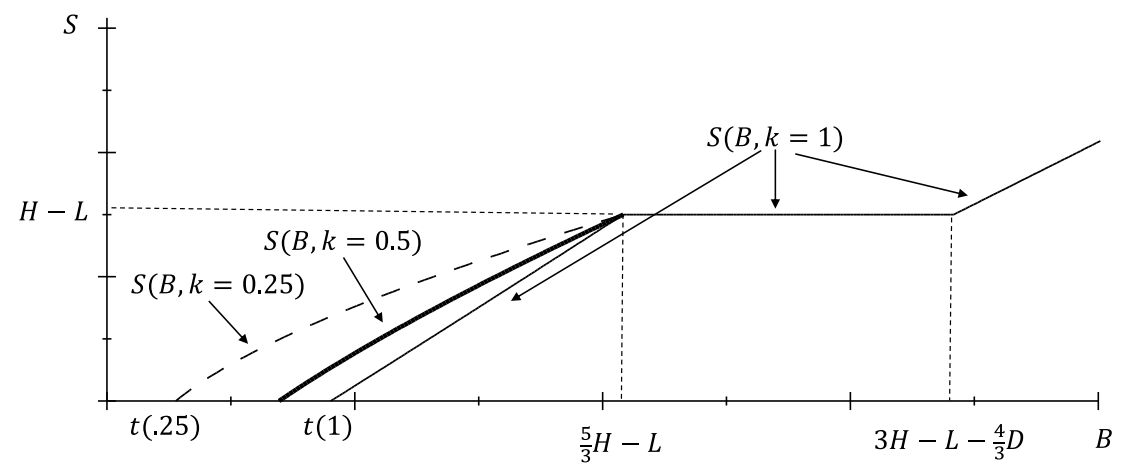

F I G U R E 7 Optimal training for different $k$

$t(k)=0$. Furthermore, the amount of training given when the solution is interior will be increased, the more weight is given to preventing erroneous selection. Proposition 5 is illustrated in Figure $7 .^{18}$

In Figure $7, S(B, k=1)$ is the total amount spent on training when $k=1$, and this is increasing in the budget for $B \in\left(t(1), \frac{5}{3} H-L\right)$ and constant at $S=H-L$ for $B \in\left[\frac{5}{3} H-L, 3 H-L-\frac{4}{3} D\right]$; in both cases, only player 2 receives training. Increases in the budget above $3 H-L-\frac{4}{3} D$ are divided between training both contestants and adding to the prize, according to Proposition 2, so that $S(B, k=1)=s_{1}+s_{2}$. Decreasing the weight $k$ to expected effort in the objective function increases training of the laggard for all interior solutions, and training starts at lower budgets. After the budget reaches $\frac{5}{3} H-L$, there is no problem of erroneous selection, since the laggard has been trained sufficiently to have the same expected ability as the opponent, and the principal uses any budget increases to increase expected effort.

\section{5 | CONCLUSION}

A contest is an often-used mechanism for eliciting effort. When contestants differ in ability or cost of effort, the incentive to provide effort is dampened, and many suggestions have been made as to how an effort-maximizing principal may level the playing field. Remedies such as giving a head start or handicap, or a bias in favor of one player, or requiring threshold levels of effort to obtain a prize are usually costless to the principal. In many real world situations, however, the principal implements a policy to redress the imbalance that has to be paid for from an existing and fixed budget. A sales manager can invest in training her employees for example, leaving a lower bonus to be granted to the "seller of the month." In human resource management more generally, weight has recently been attached to "total rewards" so that funds spent on an employer are spread over salary, bonuses and expenses to skill enhancement for instance.

We have considered the incentives of a principal to invest in skill-enhancing training that directly reduces the contest prize. Using a model with private information in which the ability distributions of the players overlap, we have shown how an effort-maximizing principal can divide her funds to increase effort through efficiency gains, even when this reduces the contest prize. The potential for realizing efficiency gains depends upon the size of the budget. If it is too small, then no training will be given at all, and all funds are channeled to the prize. Avoiding choosing the ex-post inferior player as winner gives the principal an extra incentive to train the ex-ante laggard; however, even small budgets may yield training if the cost of erroneous selection is given sufficient weight in the objective function of the principal.

Our analysis gives insight into the effect on the players' expected payoff when the principal divides her budget between the contest prize and skill-enhancement. Several effects are at work. An incremental budget unit can be used to enhance the contest prize, increasing the players' payoffs proportionately. However, using some of the increment to train the expected laggard makes the players more similar and this draws their efforts closer together; the leader increases effort whilst the laggard uses the efficiency gain to slack off. This increases the probability that the ex-ante leader wins the contest prize, but the fact that the contest prize is increased only in part, and the cost of exerting extra effort, make his payoff fall. The lower cost of effort and a lower effort level mean that the laggard has a lower probability of succeeding, but the overall effect is positive. However, it is not certain that the laggard gains a larger expected payoff 
than he would have achieved without training; this is due to the fact that training is used to increase effort, without taking account of the players' payoffs.

Two dimensions of asymmetry drive our results; the players draw ability from different intervals, and the realization is private information. In order to draw out clear conclusions we have made everything else equal, including the effect and cost of training the different players. It may well be the case, however, that it is more effective to train one of the players in the contest task; the expected leader may take training easier, and hence cost less to train, or it might be relatively cheap to help the expected laggard reach a higher skill level as he starts out low. Introducing these modifications introduces a third dimension of asymmetry and-whilst realistic—does not give clear cut results. A different base model is probably needed in order to look at these issues; one could for example dispense with the assumption of incomplete information, and look at the interplay between the known ability difference and relative training cost. We leave these issues as directions for further research.

\section{ACKNOWLEDGMENTS}

We are grateful to the Max Planck Institute for Tax Law and Public Finance in Munich, where parts of our research on this paper were done. We would like to thank Editor Stefano Barbieri and two anonymous referees for helpful comments. Errors are our own.

\section{ORCID}

Derek J. Clark (1) https://orcid.org/0000-0002-7355-4595

Tore Nilssen (i) https://orcid.org/0000-0003-2632-3855

\section{ENDNOTES}

${ }^{1}$ See Baik (1994) for an early theoretical study. Empirical studies on the discouragement effect of asymmetries are done by Sunde (2009) on tennis tournaments and Brown (2011) and Franke (2012) on golf tournaments. See also surveys by Chowdury et al. (2020), Konrad (2009), and Mealem and Nitzan (2016).

${ }^{2}$ See, for example, the survey by Chowdury et al. (2020).

${ }^{3}$ Sales-force productivity may also be affected by other costly activities undertaken by the firm that do not directly involve training. These include less administrative duties, and better access to back-room resources (see Farrell \& Hakstian, 2001).

${ }^{4}$ One branch of the literature in personnel economics focuses on cases where contestants have direct benefits from their skill levels; in the case of workers, this occurs typically because higher skills make them more attractive on the future job market, in addition to helping them to secure high compensation in the present job. See, for example, Lazear and Oyer (2012, Section 5). Here, we disregard such direct benefits from skills, instead focusing on the principal's need for balancing spending on training and compensation.

${ }^{5}$ Also other instruments have been suggested to increase efforts in asymmetric contests: Che and Gale (1998) discuss putting limits on contestants' efforts; Mealem and Nitzan (2016) discuss affecting the contestants' contest success functions and win valuations; Sisak (2009) discusses changing the prize structure; Clark and Nilssen $(2020,2021)$ discuss how to split the prize fund between early and late prizes in order to counter the effect of ex-ante differences among contestants. See more on this in the survey by Chowdhury et al. (2020).

${ }^{6}$ Other research also reports that a total leveling of the playing field would not be optimal in games among heterogeneous contestants. For example, Ryvkin (2013) discusses how the shapes of the players' effort cost functions may make it optimal to leave some heterogeneity; while Pastine and Pastine (2012) find that rectifying biases may result in a higher performance gap in a political-campaign game.

${ }^{7}$ Also here, see Chowdhury et al. (2020) for a more detailed discussion.

${ }^{8}$ In the following expressions, we have substituted $h=H-D$ and $l=L-D$.

9 The equilibrium is still valid if the ability interval of player 2 lies above that of 1 ; the player subscripts would need to be swapped in this case, since 2 is the expectedly stronger player.

${ }^{10}$ The support moves upwards so that $L^{\prime}-l^{\prime}=D$.

${ }^{11} H^{\prime \prime}-h^{\prime \prime}=D$.

${ }^{12}$ Figure 4 is generic, but $H=2, D=1$, and $L=1.25$ are used as parameter values here.

${ }^{13}$ Here, $X_{0}^{*}=\frac{L(3 H-2 D)(H+L)}{6 H^{2}} B$, as in the first line of the expression for $X^{*}$.

${ }^{14}$ In fact, when the budget is large, each contestant's expected payoff with symmetric training converges to $\frac{D}{3}$, whilst the basis payoff always increases proportionately with prize growth.

15 This is true because the square-bracketed term in Equations (16) is always nonnegative, since $L>h=H-D>\frac{(H-D)^{2}}{H}$.

${ }^{16} L>H-D>\frac{(H-D)^{3}}{H^{2}}$. 
17 This assumes that the principal does not have any other use of the budget. If the aim is to minimize the cost of erroneous selection at lowest possible cost, the principal can train the laggard to the point that $L=H$, and then give a very small prize to induce positive effort as in part (i) of Proposition 4.

${ }^{18}$ Again, this figure is generic but is drawn for parameter values $H=2, L=1.25, D=1$.

\section{REFERENCES}

Amann, E. \& Leininger, W. (1996) Asymmetric all-pay auctions with incomplete information: the two-player case. Games and Economic Behavior, 14, 1-18.

Baik, K.H. (1994) Effort levels in contests with two asymmetric players. Southern Economic Journal, 61, 367-378.

Beck-Krala, E. (2020) Total rewards. In: Idowu, S.O., et al. (Eds.) Encyclopedia of sustainable management. Springer, pp. 1-9. Available at: https://link.springer.com/content/pdf/10.1007\%2F978-3-030-02006-4_203-1.pdf

Brown, J. (2011) Quitters never win: the (adverse) incentive effects of competing with superstars. Journal of Political Economy, 119, $982-1013$.

Che, Y.K. \& Gale, I. (1998) Caps on political lobbying. American Economic Review, 88, 643-651.

Chowdhury, S.M., Esteve-Gonzalez, P. \& Mukherjee, A. (2020) Heterogeneity, leveling the playing field, and affirmative action in contests. Available at SSRN: https://ssrn.com/abstract=3655727

Clark, D.J. \& Nilssen, T. (2013) Learning by doing in contests. Public Choice, 156, 329-343.

Clark, D.J. \& Nilssen, T. (2020) Creating balance in dynamic competitions. International Journal of Industrial Organization, 69, Article 102578.

Clark, D.J. \& Nilssen, T. (2021) Competitive balance when winning breeds winners. Social Choice and Welfare, 56, 363-384.

Clark, D.J. \& Riis, C. (2001) Rank-order tournaments and selection. Journal of Economics, 73, 167-191.

Cohen, C., Kaplan, T.R. \& Sela, A. (2008) Optimal rewards in contests. RAND Journal of Economics, 39, $434-451$.

Cron, W.L., Marshall, G.W., Singh, J., Spiro, R.L. \& Sujan, H. (2005) Salesperson selection, training, and development: trends, implications, and research opportunities. Journal of Personal Selling \& Sales Management, 25, 123-136.

Farrell, S. \& Hakstian, A.R. (2001) Improving salesforce performance: a meta-analytic investigation of the effectiveness and utility of personnel selection procedures and training interventions. Psychology \& Marketing, 18, 281-316.

Franke, J. (2012) The incentive effects of levelling the playing fields: an empirical analysis of amateur golf tournaments. Applied Economics, 44, 1193-1200.

Franke, J., Leininger, W. \& Wasser, C. (2018) Optimal favoritism in all-pay auctions and lottery contests. European Economic Review, 104, $22-37$.

Fu, Q. \& Lu, J. (2009) Contest with pre-contest investment. Economics Letters, 103, 142-145.

Kirkegaard, R. (2012) Favoritism in asymmetric contests: head starts and handicaps. Games and Economic Behavior, 76, $226-248$.

Konrad, K.A. (2002) Investment in the absence of property rights: the role of incumbency advantages. European Economic Review, 46, 1521-1537.

Konrad, K.A. (2009) Strategy and dynamics in contests. Oxford University Press.

Krishnamoorthy, A., Misra, S. \& Prasad, A. (2005) Scheduling sales force training: theory and evidence. International Journal of Research in Marketing, 22, 427-440.

Lazear, E.P. \& Oyer, P. (2012) Personnel economics. In: Gibbons, R. \& Roberts, J. (Eds.) Handbook of organizational economics. Princeton University Press, pp. 479-519.

Mealem, Y. \& Nitzan, S. (2016) Discrimination in contests: a survey. Review of Economic Design, 20, 145-172.

Merton, R.K. (1968) The Matthew effect in science. Science, 159, 56-63.

Li, S. \& Yu, J. (2012) Contests with endogenous discrimination. Economics Letters, 117, 834-836.

Pastine, I. \& Pastine, T. (2012) Incumbency advantage and political campaign spending limits. Journal of Public Economics, 96, $20-32$.

Pérez-Castrillo, D. \& Wettstein, D. (2016) Discrimination in a model of contests with incomplete information about ability. International Economic Review, 57, 881-914.

Ryvkin, D. (2013) Heterogeneity of players and aggregate effort in contests. Journal of Economics and Management Strategy, 22, 728-743.

Seel, C. (2014) The value of information in asymmetric all-pay auctions. Games and Economic Behavior, 86, $330-338$.

Seel, C. \& Wasser, C. (2014) On optimal head starts in all-pay auctions. Economics Letters, 124, 211-214.

Sisak, D. (2009) Multiple-prize contests: the optimal allocation of prizes. Journal of Economic Surveys, 23, 82-114.

Sunde, U. (2009) Heterogeneity and performance in tournaments: a test for incentive effects using professional data. Applied Economics, 41, 3199-3208.

Tsoulouhas, T., Knoeber, C.R. \& Agrawal, A. (2007) Contests to become CEO: incentives, selection, and handicaps. Economic Theory, 30, 195-221. 


\section{SUPPORTING INFORMATION}

Additional supporting information may be found online in the Supporting Information section at the end of this article.

How to cite this article: Clark, D.J. \& Nilssen, T. (2021) Fatter or fitter? On rewarding and training in a contest.

Economic Inquiry, 1-20. Available from: https://doi.org/10.1111/ecin.13021

\section{A APPENDIX}

\section{A.1 | Proof of Proposition 1}

Some properties of the equilibrium outlay functions $x_{1}^{*}\left(a_{1}\right)$ and $x_{2}^{*}\left(a_{2}\right)$ are standard (see Clark \& Riis, 2001). Among these are that the effort functions have a common upper support: $x_{1}^{*}(H)=x_{2}^{*}(L)=\bar{x}$. For player $1, x_{1}^{*}(h)=0$ and $x_{1}^{*}\left(a_{1}\right)>0$ for $a_{1}>h$. For player $2, x_{2}^{*}\left(a_{2}\right)=0$ for $a_{2} \in\left[l, \widetilde{a}_{2}\right]$, implying an equilibrium effort of zero for these types.

The first-order conditions for maximizing Equations (1) and (2) are:

$$
\begin{aligned}
& \frac{g_{2}^{\prime}\left(x_{1}\right)}{D} v-\frac{1}{a_{1}}=0 ; \\
& \frac{g_{1}^{\prime}\left(x_{2}\right)}{D} v-\frac{1}{a_{2}}=0 ;
\end{aligned}
$$

where $g_{i}^{\prime}($.$) denotes the first derivative. Substituting a_{i}=g_{i}\left(x_{i}\right)$ into the first-order conditions gives a system of two differential equations:

$$
\begin{aligned}
& g_{2}^{\prime}(x) g_{1}(x)=\frac{D}{v} ; \\
& g_{1}^{\prime}(x) g_{2}(x)=\frac{D}{v} .
\end{aligned}
$$

Summing Equations (A1) and (A2) yields

$$
g_{2}^{\prime}(x) g_{1}(x)+g_{1}^{\prime}(x) g_{2}(x)=\frac{2 D}{v},
$$

with general solution

$$
g_{1}(x) g_{2}(x)=\frac{2 D}{v} x+K
$$

The constant of integration, $K$, is determined by setting $g_{1}(\bar{x})=H, g_{2}(\bar{x})=L$ into Equation (A3):

$$
\begin{aligned}
H L & =\frac{2 D}{v} \bar{x}+K \Rightarrow \\
K & =H L-\frac{2 D}{v} \bar{x} ;
\end{aligned}
$$

so that Equation (A3) becomes 


$$
g_{1}(x) g_{2}(x)=\frac{v H L-2(\bar{x}-x) D}{v} .
$$

This can then be used to substitute for $g_{2}(x)$ in the first-order condition in Equation (A1):

$$
g_{1}^{\prime}(x)-\frac{D}{v H L-2(\bar{x}-x) D^{g}} g_{1}(x)=0 .
$$

Equation (A5) has a unique solution up to a constant of integration $C$ :

$$
g_{1}(x)=\frac{1}{\sqrt{2}} C \sqrt{\frac{v H L-2(\bar{x}-x) D}{D}} .
$$

We use $g_{1}(0)=h$ to recover the constant:

$$
\begin{aligned}
g_{1}(0) & =h=\frac{1}{\sqrt{2}} C \sqrt{\frac{v H L-2 \bar{x} D}{D}} \Rightarrow \\
C & =\frac{\sqrt{2} h}{\sqrt{\frac{v H L-2 \bar{x} D}{D}}} .
\end{aligned}
$$

Thus, Equation (A6) can be written

$$
g_{1}(x)=h \sqrt{\frac{v H L-2(\bar{x}-x) D}{v H L-2 \bar{x} D}}
$$

We can use $g_{1}(\bar{x})=H$ in Equation (A7) to find $\bar{x}$ :

$$
\begin{aligned}
g_{1}(\bar{x}) & =h \sqrt{\frac{v H L}{v H L-2 \bar{x} D}} \Rightarrow \\
\bar{x} & =\frac{L\left(H^{2}-h^{2}\right)}{2 D H} v,
\end{aligned}
$$

so that we can state Equation (A7) as

$$
g_{1}(x)=\sqrt{\frac{v L h^{2}+2 D H x}{v L}},
$$

and $g_{2}(x)$ can be recovered from Equation (A4) as

$$
g_{2}(x)=\frac{\sqrt{\left(v L h^{2}+2 D H x\right) L v}}{v H} .
$$

Using $g_{i}(x)=a_{i}$ and inverting Equations (A8) and (A9) give Equations (3) and (4) in the Proposition.

\section{A.2 | Proof of Proposition 2}

Suppose the principal considers the maximization of effort in two stages. At the first stage, she sets the contest prize $v \in[0, B]$ and then, at the second stage, divides up the rest of the budget $B-v$. Working backwards, we first look at the problem of the principal when there is $S=B-v$ of the budget available for training, so that $S=s_{1}+s_{2}$. 


\section{Economic Inpuiry}

We initially make the assumption that

$$
H-L \geq S
$$

so that, even if the whole training budget goes to the laggard, he is at best ex-ante symmetric to the original leader. Substituting $s_{2}=S-s_{1}$ into Equation (7) gives the following maximization problem for the principal:

$$
\max _{s_{1} \in[0, S]} \frac{\left(L+S-s_{1}\right)\left(3 H+3 s_{1}-2 D\right)(H+L+S)}{6\left(H+s_{1}\right)^{2}} v
$$

where, for now, $v$ is treated as a constant. The maximand is decreasing in $s_{1}$ under our assumption that $\frac{H}{D}>\frac{4}{3}$. It follows that, in optimum, no training will be given to 1 , and the whole training budget will be given to $2: s_{1}=0$, and $s_{2}=S$. Inserting for $v=B-S$, this means that total expected effort is

$$
\frac{(L+S)(H+L+S)(3 H-2 D)}{6 H^{2}} v=\frac{3 H-2 D}{6 H^{2}}(L+S)(H+L+S)(B-S)
$$

So the principal's maximization problem is now

$$
\max _{S \in[0, B]} \frac{3 H-2 D}{6 H^{2}}(L+S)(H+L+S)(B-S) .
$$

The solution can be found as Equation (13), which is positive for $B>\frac{L(H+L)}{H+2 L}$.

When $B \leq \frac{L(H+L)}{H+2 L}$, total effort is falling in $S$, making it optimal to devote the whole budget to the prize as in part (i), that is, $v=B$. Furthermore, Equation (13) satisfies our condition in Equation (A10) only if the second inequality in Equation (12) holds: $B \leq \frac{5}{3} H-L$. Otherwise, it is optimal to make the players identical through training and thereafter continue training the identical players in order to solve the following problem.

We now have $H=L$, and $H-L$ of the budget already being spent on player 2 . So the maximization problem would be, from Equation (8),

$$
\max _{Z \in\left[0, \frac{B-H+L}{2}\right]}\left(H+Z-\frac{2}{3} D\right)(B-H+L-2 Z)
$$

where $Z$ is the amount spent on training each of the two contestants after they have been equalized. The optimal additional amount of training can be determined as $Z^{*}=\frac{1}{12}(3 B-9 H+3 L+4 D)$, which is positive for $B>3 H-L-\frac{4}{3} D$. Inserting $Z^{*}$ into the expression for total training, $S=H-L+2 Z^{*}$, we get Equation (14) in part (iv).

When $B \leq 3 H-L-\frac{4}{3} D$, the principal will not train the players once symmetry is reached, since $Z^{*} \leq 0$; hence, in part (iii), $S=H-L$, and the prize is $B-H+L$.

\section{A.3 | Proof of Proposition 3}

(i) Calculation shows that $\frac{\partial \rho^{*}}{\partial L}>0$ for $\frac{H^{2}+(H-D)^{2}}{2 H}>L$. Recalling that $L>\max \{H-D, D\}$, we have to check whether the interval

$$
L \in\left(\max \{H-D, D\}, \frac{H^{2}+(H-D)^{2}}{2 H}\right)
$$

is well defined, that is, whether $\frac{H^{2}+(H-D)^{2}}{2 H}>\max \{H-D, D\}$. 
(a) Assume first that $H-D>D$, that is, $H>2 D$. Then $\frac{H^{2}+(H-D)^{2}}{2 H}-(H-D)=\frac{1}{2} \frac{D^{2}}{H}>0$, and the interval is well defined in this case.

(b) Assume next that $D>H-D$, that is, $2 D>H$. Then $\frac{H^{2}+(H-D)^{2}}{2 H}-D=\frac{1}{2} \frac{-4 H D+D^{2}+2 H^{2}}{H}>0$ for $H>\left(1+\frac{1}{\sqrt{2}}\right) D$.

Combining (a) and (b) gives the result in part (i). If $H>\left(1+\frac{1}{\sqrt{2}}\right) D$, then we have $\frac{\partial \rho^{*}}{\partial L}>0$ for $L \in\left(\max \{H-D, D\}, \frac{H^{2}+(H-D)^{2}}{2 H}\right)$. If $\frac{4 D}{3}<H<\left(1+\frac{1}{\sqrt{2}}\right) D$, or $L>\frac{H^{2}+(H-D)^{2}}{2 H}$, then we have $\frac{\partial \rho^{*}}{\partial L}<0$.

(ii) Calculation gives $\frac{\partial \rho^{*}}{\partial H}=\frac{1}{2} \frac{2 H^{2}(L+D-H)-L D^{2}}{H^{2} D^{2}}$, which is positive when evaluated at $H=L$, and negative at $H=L+D$. Furthermore, $\frac{\partial^{2} \rho^{*}}{\partial H^{2}}=-\frac{H^{3}-L D^{2}}{H^{3} D^{2}}<0$, and the only real root of $2 H^{2}(L+D-H)-L D^{2}=0$ can be calculated as

$$
\begin{aligned}
& \widehat{H}=3[3 \sigma+(L+D)]+\frac{(L+D)^{2}}{\sigma}, \text { where } \\
& \sigma=\frac{1}{6} \sqrt[3]{6 \sqrt{3} D \sqrt{L\left[8\left(L^{3}-D^{3}\right)-3 D L(8 L-D)\right]}+(L+4 D)(2 L-D)^{2}} .
\end{aligned}
$$

\section{A.4 | Proof of Lemma 1}

(i) Calculation gives $\frac{\partial \Gamma^{*}}{\partial L}<0$ for $L>\frac{3 H^{3}-2 D^{3}-6 H D(H-D)}{3 H^{2}}$, and this is least likely to hold for low values of $L$. Recall that the lowest $L$ is $\max \{H-D, D\}$. We prove our claim in two steps.

Step 1. Assume first that $H-D>D$, that is, that $H>2 D$. We need to check that $H-D>\frac{3 H^{3}-2 D^{3}-6 H D(H-D)}{3 H^{2}}$, which implies that $3 H^{2}-6 H D+2 D^{2}>0$. Given that $H>\frac{4 D}{3}, 3 H^{2}-6 H D+2 D^{2}>0$ is satisfied for $H>\left(\frac{1}{\sqrt{3}}+1\right) D$, which must be true, since $H>2 D>\left(\frac{1}{\sqrt{3}}+1\right) D$.

Step 2. Assume next that $D>H-D$, that is, that $2>\frac{H}{D}\left(>\frac{4}{3}\right)$. We must show that

$$
D>\frac{3 H^{3}-2 D^{3}-6 H D(H-D)}{3 H^{2}}
$$

which can be shown to hold for $\zeta:=-3 H^{3}+3 H D(3 H-2 D)+2 D^{3}>0$. It can be determined that $\zeta=0$ has one real positive solution given by

$$
\tilde{H}=D\left(1+\frac{1}{3} \sqrt[3]{3 \sqrt{6}+9}+\frac{1}{\sqrt[3]{3 \sqrt{6}+9}}\right) \approx 2.24 D
$$

and that $\zeta>0$ if and only if $H<\tilde{H}$, which always holds under our assumption in Step 2 that $\frac{H}{D}<2$.

It follows from Steps 1 and 2 that $\Gamma^{*}$ is monotonically decreasing in $L$ for all $L \in(\max \{H-D, D\}, H]$.

(ii) Calculation shows that $\frac{\partial \Gamma^{*}}{\partial H}>0$ for $L>\frac{3 H^{2}(H-D)^{2}}{3 H^{3}-3 H D^{2}+2 D^{3}}$, which is least likely to hold for low values of $L$. Note that, if $L=H-D>D$, then $\frac{\partial \Gamma^{*}}{\partial H}>0$ if $H-D>\frac{3 H^{2}(H-D)^{2}}{3 H^{3}-3 H D^{2}+2 D^{3}}$; this holds for $3 H(H-D)+2 D^{2}>0$, which is always true.

If $L=D>H-D$, then $\frac{\partial \Gamma^{*}}{\partial H}>0$ requires

$$
D>\frac{3 H^{2}(H-D)^{2}}{3 H^{3}-3 H D^{2}+2 D^{3}}=\frac{3 H^{2}(H-D)^{2}}{3 H(H-D)(H+D)+2 D^{3}} .
$$

This implies $3 H D(H-D)(H+D)+2 D^{4}>3 H^{2}(H-D)^{2}$, which can be rearranged to give

$$
3 H(H-D)[D(H+D)-H(H-D)]+2 D^{4}>0 .
$$




\section{0 _ Economic Inpuiry}

The term in square brackets is positive, since $H+D>H$, and $D>H-D$ for this case, and thus the inequality holds. Hence $\frac{\partial \Gamma^{*}}{\partial H}>0$ always.

\section{A.5 | Proof of Proposition 5}

Letting $S$ denote training given to the laggard, the principal chooses $S$ to maximize

$$
\begin{aligned}
\Omega^{*}(k)= & k \frac{(L+S)(3 H-2 D)(H+L+S)}{6 H^{2}}(B-S) \\
& -(1-k) \frac{(H-L-S)^{2}\left[H^{2}(L+S)-(H-D)^{3}\right]}{6 H^{2} D^{2}} \\
= & k X^{*}(S, B, H, L, D)-(1-k) \Gamma^{*}(S, H, L, D) .
\end{aligned}
$$

The first-order condition for an interior maximum is

$$
k \frac{\partial X^{*}}{\partial S}-(1-k) \frac{\partial \Gamma^{*}}{\partial S}=0
$$

with second-order condition

$$
\frac{\partial^{2} \Omega^{*}}{\partial S^{2}}<0
$$

Total differentiation in Equation (A11) with respect to $S$ and $k$ gives

$$
\left(\frac{\partial X^{*}}{\partial S}+\frac{\partial \Gamma^{*}}{\partial S}\right) d k+\frac{\partial^{2} \Omega^{*}}{\partial S^{2}} d s=0
$$

which, using Equation (A11), can be written as

$$
\frac{d S}{d k}=\frac{-\frac{\partial \Gamma^{*}}{\partial S}}{k \frac{\partial^{2} \Omega^{*}}{\partial S^{2}}}<0
$$

where the sign of the numerator is positive from Lemma 1 , since $\operatorname{sign}\left(\frac{\partial \Gamma^{*}}{\partial S}\right)=\operatorname{sign}\left(\frac{\partial \Gamma^{*}}{\partial L}\right)$, and the denominator is negative from Equation (A12).

To see that $\frac{d t}{d k}>0$ when $t(k)>0$, consider how $S$ responds to a change in the budget in the range $S \in(0, H-L)$. From Equation (A11), we have

$$
\frac{d S}{d B}=-\frac{k \frac{\partial X^{*}}{\partial B \partial S}}{\frac{\partial^{2} \Omega^{*}}{\partial S^{2}}}>0,
$$

since $\frac{\partial X^{*}}{\partial B \partial S}=\frac{(3 H-2 D)(H+2 L+2 S)}{6 H^{2}}>0$ and Equation (A12) holds. Now, fix a $B=t(k)$ such that $S(t(k), k)=0$. Compare this with some $k^{\prime}<k$, which, since $\frac{d S}{d k}<0$, implies $S\left(t(k), k^{\prime}\right)>S(t(k), k)=0$. Since $S$ is increasing in $B$, by Equation (A13), it follows that $S\left(t\left(k^{\prime}\right), k^{\prime}\right)=0$ for $t\left(k^{\prime}\right)<t(k)$, and hence $\frac{d t}{d k}>0$. 